\title{
DEVELOPMENT OF A GENERAL COMPUTATION ALGORITHM FOR THE ANALYSIS OF MMIC STRUCTURES BASED ON A THREE-DIMENSIONAL MODEL
}

\author{
R. MAKRI*, M. GARGALAKOS and N. K. UZUNOGLU \\ Institute of Communications and Computer Systems, National Technical University of Athens, \\ 9, Heroon Polytechniou Str. Zografou Athens 15773, Greece
}

\begin{abstract}
A generalized three-dimensional computational numerical code is developed for Monolithic Microwave Integrated Circuit (MMIC) structures based on a full wave approach using integral equation techniques. The electromagnetic properties of the MMIC structures are calculated using a Method of Moments - Galerkin technique based on integral equations and the relevant Green function to obtain a rigorous formulation. The MMIC structures are assumed to have an arbitrary geometry involving orthogonal parallelepiped 'cells', each one of which is characterized with finite permittivity and conductivity values. A novel approach is proposed to treat the incident: reflected and transmitted waves at the two microstrip lines, which stand as input and output of the circuit. Their current distribution is also being taken into account in terms of incident, reflected and transferred waves. The derived matrix is being inverted and the obtained results are the unknown coefficients of the plane waves inside the 'cells' and also the reflection $R$ and transfer $T$ coefficients. The derived numerical results concern linear cases, while the examination of non-linear structures have been taken into account in the analytical formulation.
\end{abstract}

Keywords: MMIC circuits; 2D linear and non-linear analysis; Galerkin technique; Wiener-Voltera Method

\section{INTRODUCTION}

The continuous applications of microwave and millimeter-wave integrated circuits in microstrip and packaging technology have resulted to an emerging research interest about the analysis of layered electromagnetic structures. Following this fact, the development of CAD tools for microwave structures based on accurate numerical techniques is imposed. The traditional circuit theory based on lumped or even distributed parameter element modeling seems to be inadequate for modern applications especially at frequencies exceeding $30 \mathrm{GHz}$ [1-3]. Thus, as a main problem arise the calculation of the effects related to both the dielectric and conducting parts of three dimension geometries.

At the present work a general computation algorithm to treat three-dimensional integrated circuit structures is proposed. The MMIC structures under consideration are assumed to be in their most general form. In other words, they are three dimensional, finite at size and are characterized with finite permittivity and conductivity values. This geometrical approach is the one that expresses in the most possible way the MMIC structural form often met in practice. Thus, the MMIC structures are assumed to have an arbitrary geometry involving

* Corresponding author. E-mail: rodia@esd.ece.ntua.gr 
orthogonal parallelepiped "cells", each of this is characterized with finite permittivity and conductivity values as stated above. In order for this approach to be applied properly and in such a way that most "physical" structures are encountered, a reference structure is shown in Figure 1 representing the most general form.

A detailed inspection of the geometry is given in the following. As it is seen from Figure 1, is assumed that the whole structure is placed over a two-layer substrate. This would resemble the GaAs and $\mathrm{SiAl}$ layers, which are the most used substrates in popular libraries (such as the GEC-Marconi library supported by Libra Touchstone HP-Eesof). The substrate layers are characterized also with finite permittivity and conductivity values which can be expressed as the complex relative permittivities $\dot{\varepsilon}_{r s 1}$ and $\dot{\varepsilon}_{r s 2}$. This two-layer substrate is grounded on the bottom side where the system of $x-y-z$ coordinates originates. Following this, the $z=0$ plane is the ground plane while at the $z=D=d_{1}+d_{2}$ plane the orthogonal parallelepiped cells are placed forming the MMIC structures.

The medium above the two layered substrate (that is, above $z=D$ plane) and outside of the circuit structure is taken to be free space characterized with the free space permittivity $\varepsilon_{0}=\left(10^{-9} /(36 \pi)\right)(F / m)$ and magnetic permeability $\mu=\mu_{0}=4 \pi \times 10^{-7}(\mathrm{H} / \mathrm{m})$. The magnetic permeability $\mu_{0}$ also characterizes the whole space; all structures and layers are assumed to have the same $\mu_{0}$.

Above the double layer substrate, a circuit structure consisting of orthogonal parallelepiped brick shape cells with various dimensions and properties is present, forming the geometry under consideration. The electromagnetic properties of each unit cell are characterized by finite values of permittivity and conductivity, or in other words, by the homogeneous complex relative permittivity value $\varepsilon_{r_{i}}=\varepsilon_{r_{i}}-j\left(\sigma_{i} / \omega \varepsilon_{0}\right)\left(\varepsilon_{r_{i}}\right.$ and $\sigma_{i}$ being the real permittivity and conductivity value). The specific cell is denoted by the cell index $i$, while $\omega$ is the angular radiation frequency and $\varepsilon_{0}$ is the free space permittivity as above. Furthermore, the center of gravity of the $i$ th cell is given by $\left(x_{0}^{i}, y_{0}^{i}, z_{0}^{i}\right)$. As it is known the propagation constant in a medium with such electromagnetics properties is given by

$$
k=\omega \sqrt{\varepsilon_{0} \mu_{0}} \cdot \sqrt{\varepsilon_{r} \cdot\left(1-j \frac{\sigma}{\omega \varepsilon_{0}}\right)}
$$

or $\left.k=\omega \sqrt{\varepsilon_{0} \mu_{0}} \cdot \sqrt{\varepsilon_{r} \cdot(1+j \tan \delta}\right), \quad \tan \delta$ given by $\tan \delta=-\frac{\sigma}{\omega \varepsilon_{0}}$

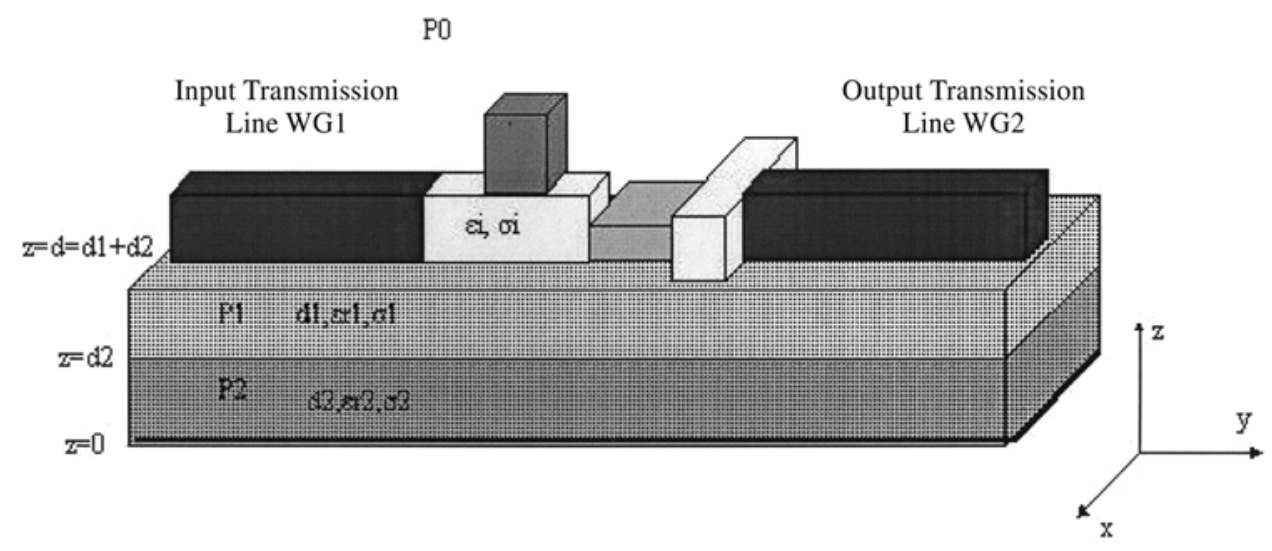

FIGURE 1 General circuit structure 
As shown from Figure 1 the whole structure is driven by two microstrip lines which act as input and output of the circuit, assumed to be half-infinite in length, while their current distribution is being taken into account in terms of incident, reflected and transferred waves. Each strip over the grounded two layered substrate consists of infinite conductivity due to the perfect conductor assumption. Thus, the assumption includes diminishingly thin strips where the current surface density substitutes the electric field (which is zero inside the strip). In the whole analysis that follows, an $e^{+j \omega t}$ time dependence is assumed for all field quantities.

\section{FORMULATION}

In order to define the unknown electric field and thus to characterize exactly all the electromagnetic properties of the above structure an integral equation approach is implemented. Starting from the Maxwell equations applied in the specific geometry of the orthogonal parallelopiped cells, the mathematical formulation of the electromagnetic field in an integral form is derived in the Po space where the cells represent structure's sources of excitation. Using the Maxwell equations, the well-known Helmholtz equation is derived

$$
\nabla^{2} \bar{E}(\bar{r})+k^{2} \bar{E}(\bar{r})=j \omega \mu_{v} \bar{J}(\bar{r})
$$

where $\bar{J}(\bar{r})$ is the current distribution. The above equation is valid in every different volume with specific electromagnetic characteristics under consideration, respectively. The boundary conditions along with the degradation of the radiated waves must be applied and the cells are considered as perturbation of the Po's space dielectric constant. Thus

$$
\nabla x \bar{H}(\bar{r})-j \omega \varepsilon_{0} \bar{E}(\bar{r})=j \omega\left(\varepsilon(\bar{r})-\varepsilon_{0}\right) \bar{E}(\bar{r})
$$

where an equivalent current distribution $\bar{J}^{\delta}(\bar{r})$ can be extracted which satisfying the

$$
\bar{J}^{\delta}(\bar{r})=j \omega\left(\varepsilon(\bar{r})-\varepsilon_{0}\right) \bar{E}(\bar{r})=j \omega \varepsilon_{v} \delta \varepsilon_{r}(\bar{r}) \bar{E}(\bar{r})
$$

By that way, the perturbations $\delta \varepsilon_{r}(\bar{r})$ in the dielectric constant $\varepsilon_{0}$ due to the orthogonal cells correspond to an equivalent current source $\bar{J}^{\delta}(\bar{r})$ with bias current characteristics and dependent from the stimulated unknown electric field $\bar{E}(\bar{r})$ inside the cells. The solution of the above equations is very well treated in bibliography $[4,5]$ ending to an integral equation based on the dyadic Green function for the electric field

$$
\bar{E}(\bar{r})=-j \omega \mu_{v} \iint_{V} \int_{\bar{G}}\left(\bar{r}, \bar{r}^{\prime}\right) \cdot \bar{J}^{\delta}\left(\bar{r}^{\prime}\right) \mathrm{d} V\left(\bar{r}^{\prime}\right)
$$

which can be written also as

$$
\bar{E}(\bar{r})=k_{o}^{2} \iiint_{V} \overline{\bar{G}}\left(\bar{r}, \bar{r}^{\prime}\right) \cdot \delta \varepsilon_{r}\left(\bar{r}^{\prime}\right) \bar{E}\left(\bar{r}^{\prime}\right) \mathrm{d} V\left(\bar{r}^{\prime}\right)
$$

where $\bar{E}_{j}\left(\bar{r}_{j}\right)$ is the unknown electric field calculated at the observation points and $\bar{E}_{i}\left(\bar{r}_{i}^{\prime}\right)$ is the electric field created by the field sources. Equation (6) stands as the basic integral equation for the formulation of the present analysis. Before proceeding further the dyadic Green's function must be described. 


\section{Green's Function}

Due to arbitrary circuit's geometry used, Eq. (6) is a general one and can be applied in all geometries along with layered structures, since no particular assumption of the placement of the cells is made. On that basis, a Green function depending on the specific two-layered structure must be computed. The origin for the computation of layered structures Green functions can be found in bibliography [6], while for the present analysis the dyadic Green function has been already derived [7] and is given in the following for the completeness of the analysis.

$$
\overline{\bar{G}}\left(\bar{r}, \bar{r}^{\prime}\right)=\frac{1}{(2 \pi)^{2}} \int_{-\infty}^{\infty} \int \overline{\bar{g}}\left(k_{x}, k_{y} ; z, z^{\prime}\right) e^{j\left(k_{x}\left(x-x^{\prime}\right)+k_{y}\left(y-y^{\prime}\right)\right)} \mathrm{d} k_{x} \mathrm{~d} k_{y}
$$

where

$$
\overline{\bar{g}}=\overline{\bar{g}}^{\mathrm{sec}}+\overline{\bar{g}}^{\mathrm{pr}}
$$

$\overline{\bar{g}}^{\mathrm{sec}}$ and $\overline{\bar{g}}^{\mathrm{pr}}$ being the primary and secondary term respectively and $\bar{r}_{j}$ is the observation vector, $\bar{r}_{i}^{\prime}$ is the source vector. The above relations can be written as

$$
\begin{aligned}
& \overline{\bar{g}}^{\mathrm{sec}}=\overline{\bar{g}}_{1} \cdot e^{-j k_{z o}\left(z+z^{\prime}\right)} \\
& \overline{\bar{g}}^{\mathrm{pr}}=\overline{\bar{g}}_{2} \cdot e^{j k_{z o}\left(z-z^{\prime}\right)} \cdot u\left(z^{\prime}-z\right)+\overline{\bar{g}}_{3} \cdot e^{-j k_{z o}\left(z-z^{\prime}\right)} \cdot u\left(z-z^{\prime}\right)
\end{aligned}
$$

The terms $\overline{\bar{g}}_{1}, \overline{\bar{g}}_{2}, \overline{\bar{g}}_{3}$ can be found in their analytical form in [7].

Equation (7) can be written in the following complete form in order to include also the case where the observation point is within the field source volumes,

$$
\overline{\bar{G}}\left(\bar{r}, \bar{r}^{\prime}\right)=\frac{1}{(2 \pi)^{2}} \int_{-\infty}^{\infty} \int \overline{\bar{g}}\left(k_{x}, k_{y} ; z, z^{\prime}\right) e^{j\left(k_{x}\left(x-x^{\prime}\right)+k_{y}\left(y-y^{\prime}\right)\right)} \mathrm{d} k_{x} \mathrm{~d} k_{y}-\frac{\hat{z} \hat{z}}{k_{o}^{2}} \delta\left(\bar{r}-\bar{r}^{\prime}\right)
$$

in other words, that includes the singularity $\bar{r}=\bar{r}^{\prime}$.

\section{INTEGRAL EQUATION}

Following the previous analysis and assuming that the general form of the unknown electric field [8] is given by

$$
\bar{E}(\bar{r})=\bar{e}(\bar{r}) e^{-j \beta y}
$$

(where $\bar{e}(\bar{r})$ denotes the transverse distribution of the electric field in the $x$-z layer while the parameter $\beta$ is the propagation constant). The following integral equation is then derived

$$
\begin{aligned}
\left(\overline{\overline{1}}+\frac{k_{V}^{2}}{k_{o}^{2}} \delta \varepsilon_{r_{j}} \hat{z} \hat{z}\right) \cdot \bar{e}_{j}(\bar{r})= & \frac{k_{V}^{2}}{2 \pi} \sum_{i=1}^{N} \delta \varepsilon_{r_{i}} \iiint_{V_{i}} \mathrm{~d} x^{\prime} \mathrm{d} y^{\prime} \mathrm{d} z^{\prime} \\
& \times\left(\int_{-\infty}^{\infty} \int \mathrm{d} k_{x} \mathrm{~d} k_{y} e^{j\left(k_{x}\left(x-x^{\prime}\right)+k_{y}\left(y-y^{\prime}\right)\right)} \overline{\bar{g}}\left(k_{x}, k_{y}^{\prime} ; z, z^{\prime}\right)\right) \cdot \bar{e}_{i}(\bar{r})
\end{aligned}
$$


The analysis assumes that each elementary cell and the two input and output transmission lines can both be considered as observation points and field sources. Therefore, the unknown electric field calculated in a specific elementary cell, is the sum of the contribution of each source, including the observation cell itself. By that way the electric field of the $j$-cell at the position $\bar{r}$ (observation point) is, in fact, the sum of the contributions of the $i$-cells (field sources) at position $\bar{r}^{\prime}$. Due to the fact that the perturbations are generated in the volumes, which present electrical characteristics different from the free space, it is obvious that, the total electric field occurs from the sum of the contributions of the fields generated by the orthogonal parallelepiped cells which "disturb" the space Po.

This equation is applied for the total number $N$ of the cells that is for $j=1,2, \ldots, N$ where a system of $N$ integral equations is then ensued with unknown coefficients the distributions of the electric fields in the volumes $V_{j}$ of the $N$ cells. The input and output transmission lines are also assumed as cells (with half infinite dimension along $y$ axis) with $e_{r}=1$ as dielectric permittivity and infinite conductivity since they are perfect conductors [9]. Thus the derived system includes $N+2$ equations providing the electric field in all volumes and assuming as filed sources the $N$ cells and the input and output transmission lines.

$$
\begin{aligned}
\bar{E}(\bar{r}) & =k_{V}^{2} \sum_{i=1}^{N+2} \delta \varepsilon_{r_{i}} \iiint_{V_{i}} \overline{\bar{G}}\left(\bar{r}, \bar{r}^{\prime}\right) \cdot \bar{E}\left(\bar{r}^{\prime}\right) \mathrm{d} V\left(\bar{r}^{\prime}\right) \\
& =\iiint_{\text {everyvolume }} k_{o}^{2}\left[\delta \varepsilon_{r_{i}} \sum_{i=1}^{N} \overline{\bar{G}}\left(\bar{r}, \bar{r}^{\prime}\right) \cdot \bar{E}_{\text {cells }}\left(\bar{r}^{\prime}\right)+\overline{\bar{G}}\left(\bar{r}, \bar{r}^{\prime}\right) \cdot \bar{J}_{W G 1}\left(\bar{r}^{\prime}\right)+\overline{\bar{G}}\left(\bar{r}, \bar{r}^{\prime}\right) \cdot \bar{J}_{W G 2}\left(\bar{r}^{\prime}\right)\right] \mathrm{d} V\left(\bar{r}^{\prime}\right)
\end{aligned}
$$

where,

$\bar{E}_{\text {cells }}\left(\bar{r}^{\prime}\right)$ is the field ought to the contribution of the $N$ cells

$\bar{J}_{W G 1}\left(\bar{r}^{\prime}\right)$ is the current distribution from the input transmission line and

$\bar{J}_{W G 2}\left(\bar{r}^{\prime}\right)$ is the current distribution from the output transmission line since the latter considered as perfect conductors.

Assuming that the incident current wave has a cosine dependence regarding variable $x$ its form [10] is the following

$$
\bar{J}_{o}^{>\pi}\left(r^{\prime}\right)=\hat{y} J_{o} \cos \left(\frac{\pi x}{\alpha^{\prime}}\right) e^{-j \beta y}
$$

where $J_{o}$ is the wave amplitude which for the sake of simplicity is assumed to be unity. In relevance the reflected current is

$$
\bar{J}_{o}^{<\alpha}\left(r^{\prime}\right)=\hat{y} R J_{o} \cos \left(\frac{\pi x}{\alpha^{\prime}}\right) e^{j \beta y}
$$

where $R$ is the unknown reflection coefficient and the transmitted current is

$$
\bar{J}_{o}^{>\mu}\left(r^{\prime}\right)=\hat{y} T J_{o} \cos \left(\frac{\pi x}{\alpha^{\prime}}\right) e^{-j \beta y}
$$

where $T$ is the unknown transmission coefficient respectively. 
From all the above the final integral formulation that describes the circuitry is given by the following equation

$$
\begin{aligned}
\bar{E}(\bar{r})=\iiint_{\text {everyvolume }} & {\left[k_{o}^{2} \delta \varepsilon_{r_{i}} \sum_{i=1}^{N} \overline{\bar{G}}\left(\bar{r}, \bar{r}^{\prime}\right) \cdot \bar{E}_{\text {cells }}\left(r^{\prime}\right)+\overline{\bar{G}}\left(\bar{r}, \bar{r}^{\prime}\right) \cdot\left(\bar{J}_{o}^{>\pi}\left(r^{\prime}\right)+R \bar{J}_{o}^{<\alpha}\left(r^{\prime}\right)\right)\right.} \\
& \left.+\overline{\bar{G}}\left(\bar{r}, \bar{r}^{\prime}\right) \cdot T \bar{J}_{o}^{>\mu}\left(r^{\prime}\right)\right] \mathrm{d} V\left(\bar{r}^{\prime}\right)
\end{aligned}
$$

Considering the possible positions of the observation vector $\bar{r}$ in the circuitry under consideration the following cases are examined:

Case $A$ where the orthogonal cells are assumed as observation points. From Eq. (18) the derived new equation is

$$
\begin{gathered}
\bar{E}(\bar{r})=\iiint_{\text {everyvolume }}[ \\
{\left[k_{o}^{2} \delta \varepsilon_{r_{i}} \sum_{i=1}^{N} \overline{\bar{G}}\left(\bar{r}, \bar{r}^{\prime}\right) \cdot \bar{E}_{\text {cells }}\left(r^{\prime}\right)+\overline{\bar{G}}\left(\bar{r}, \bar{r}^{\prime}\right) \cdot\left(\bar{J}_{o}^{>\pi}\left(r^{\prime}\right)+R \bar{J}_{o}^{<\alpha}\left(r^{\prime}\right)\right)\right.} \\
\left.+\overline{\bar{G}}\left(\bar{r}, \bar{r}^{\prime}\right) \cdot T \bar{J}_{o}^{>\mu}\left(r^{\prime}\right)\right] \mathrm{d} V\left(\bar{r}^{\prime}\right)
\end{gathered}
$$

which equals to

$$
\begin{aligned}
\left(\overline{\overline{1}}+\frac{k_{V}^{2}}{k_{o}^{2}} \delta \varepsilon_{r_{j}} \hat{z} \hat{z}\right) \cdot & \bar{e}_{j}(\bar{r}) \\
=\iiint \int_{\text {everyvolume }}[ & \frac{k_{o}^{2}}{2 \pi} \sum_{i=1}^{N} \delta \varepsilon_{r_{i}}\left(\int_{-\infty}^{\infty} \int \mathrm{d} k_{x} \mathrm{~d} k_{y} e^{j\left(k_{x}\left(x-x^{\prime}\right)+k_{y}\left(y-y^{\prime}\right)\right)} \overline{\bar{g}}\left(k_{x}, k_{y}^{\prime} ; z, z^{\prime}\right)\right) \cdot \bar{e}_{i}\left(\overline{\boldsymbol{r}}^{\prime}\right) \\
& +\left(\int_{-\infty}^{\infty} \int \mathrm{d} k_{x} \mathrm{~d} k_{y} e^{j\left(k_{x}\left(x-x^{\prime}\right)+k_{y}\left(y-y^{\prime}\right)\right)} \overline{\bar{g}}\left(k_{x}, k_{y}^{\prime} ; z, z^{\prime}\right)\right) \cdot\left(\bar{J}_{o}^{>\pi}\left(\bar{r}^{\prime}\right)+\bar{J}_{o}^{<\alpha}\left(\bar{r}^{\prime}\right)\right) \\
& \left.+\left(\int_{-\infty}^{\infty} \int \mathrm{d} k_{x} \mathrm{~d} k_{y} e^{j\left(k_{x}\left(x-x^{\prime}\right)+k_{y}\left(y-y^{\prime}\right)\right)} \overline{\bar{g}}\left(k_{x}, k_{y}^{\prime} ; z, z^{\prime}\right)\right) \cdot \bar{J}_{o}^{>\mu}\left(\bar{r}^{\prime}\right)\right] \mathrm{d} x^{\prime} \mathrm{d} y^{\prime} \mathrm{d} z^{\prime}
\end{aligned}
$$

Case $B$ where the input transmission line serves as observation volume. Then the derived equation is the following

$$
\begin{gathered}
\bar{E}_{W G 1}(\bar{r})=\iiint_{\text {everyvolume }}[ \\
{\left[k_{o}^{2} \delta \varepsilon_{r_{i}} \sum_{i=1}^{N} \overline{\bar{G}}\left(\bar{r}, \bar{r}^{\prime}\right) \cdot \bar{E}_{\text {cells }}\left(r^{\prime}\right)+\overline{\bar{G}}\left(\bar{r}, \bar{r}^{\prime}\right) \cdot\left(\bar{J}_{o}^{>\pi}\left(r^{\prime}\right)+R \bar{J}_{o}^{<\alpha}\left(r^{\prime}\right)\right)\right.} \\
\left.+\overline{\bar{G}}\left(\bar{r}, \bar{r}^{\prime}\right) \cdot T \bar{J}_{o}^{>\mu}\left(r^{\prime}\right)\right] \mathrm{d} V\left(\bar{r}^{\prime}\right)
\end{gathered}
$$


which results in the following equation due to the fact that the line is perfect conductor

$$
\begin{aligned}
0=\iiint_{\text {everyvolume }}[ & \frac{k_{o}^{2}}{2 \pi} \sum_{i=1}^{N} \delta \varepsilon_{r_{i}}\left(\int_{-\infty}^{\infty} \int \mathrm{d} k_{x} \mathrm{~d} k_{y} e^{j\left(k_{x}\left(x-x^{\prime}\right)+k_{y}\left(y-y^{\prime}\right)\right)} \overline{\bar{g}}\left(k_{x}, k_{y}^{\prime} ; z, z^{\prime}\right)\right) \cdot \bar{e}_{i}\left(\bar{r}^{\prime}\right) \\
& +\left(\int_{-\infty}^{\infty} \int \mathrm{d} k_{x} \mathrm{~d} k_{y} e^{j\left(k_{x}\left(x-x^{\prime}\right)+k_{y}\left(y-y^{\prime}\right)\right)} \overline{\bar{g}}\left(k_{x}, k_{y}^{\prime} ; z, z^{\prime}\right)\right) \cdot\left(\bar{J}_{o}^{>\pi}\left(\bar{r}^{\prime}\right)+\bar{J}_{o}^{<\alpha}\left(\bar{r}^{\prime}\right)\right) \\
& \left.+\left(\int_{-\infty}^{\infty} \int \mathrm{d} k_{x} \mathrm{~d} k_{y} e^{j\left(k_{x}\left(x-x^{\prime}\right)+k_{y}\left(y-y^{\prime}\right)\right)} \overline{\bar{g}}\left(k_{x}, k_{y}^{\prime} ; z, z^{\prime}\right)\right) \cdot \bar{J}_{o}^{>\mu}\left(\bar{r}^{\prime}\right)\right] \mathrm{d} x^{\prime} \mathrm{d} y^{\prime} \mathrm{d} z^{\prime}
\end{aligned}
$$

Case C Respectively to case B when the output transmission line is the observation point

$$
\begin{aligned}
0=\iiint_{\text {everyvolume }}[ & \frac{k_{o}^{2}}{2 \pi} \sum_{i=1}^{N} \delta \varepsilon_{r_{i}}\left(\int_{-\infty}^{\infty} \int \mathrm{d} k_{x} \mathrm{~d} k_{y} e^{j\left(k_{x}\left(x-x^{\prime}\right)+k_{y}\left(y-y^{\prime}\right)\right)} \overline{\bar{g}}\left(k_{x}, k_{y}^{\prime} ; z, z^{\prime}\right)\right) \cdot \bar{e}_{i}\left(\bar{r}^{\prime}\right) \\
& +\left(\int_{-\infty}^{\infty} \int \mathrm{d} k_{x} \mathrm{~d} k_{y} e^{j\left(k_{x}\left(x-x^{\prime}\right)+k_{y}\left(y-y^{\prime}\right)\right)} \overline{\bar{g}}\left(k_{x}, k_{y}^{\prime} ; z, z^{\prime}\right)\right) \cdot\left(\bar{J}_{o}^{>\pi}\left(\bar{r}^{\prime}\right)+\bar{J}_{o}^{<\alpha}\left(\bar{r}^{\prime}\right)\right) \\
& \left.+\left(\int_{-\infty}^{\infty} \int \mathrm{d} k_{x} \mathrm{~d} k_{y} e^{j\left(k_{x}\left(x-x^{\prime}\right)+k_{y}\left(y-y^{\prime}\right)\right)} \overline{\bar{g}}\left(k_{x}, k_{y}^{\prime} ; z, z^{\prime}\right)\right) \cdot \bar{J}_{o}^{>\mu}\left(\bar{r}^{\prime}\right)\right] \mathrm{d} x^{\prime} \mathrm{d} y^{\prime} \mathrm{d} z^{\prime}
\end{aligned}
$$

From all the three cases under consideration a system of $N+2$ equations is being developed in the following way: $N$ equations (from the $N$ cells) are produced from case A while the 2 last equations are provided from case $\mathrm{B}$ and $\mathrm{C}$ respectively.

\section{RESOLVING THE EQUATIONS - GALERKIN TECHNIQUE}

In order the system to be solved a Method of Moments - Galerkin technique is being implemented. The method presupposes a selection of entire domain basic functions necessary for the field description in every element of the circuitry. Using the Galerkin technique the system of integral equations is converted to an algebraic linear non-homogenous one $[11,12]$. To this end both the unknown electric field and the field created by the sources are expanded in terms of piecewise basic functions being a superposition of plane waves as

$$
\bar{e}_{i}(\bar{r}) \approx \sum_{m=1}^{M_{i}} \sum_{n=1}^{N_{i}} \bar{c}_{i m n} f_{i m n}(\bar{r})=\sum_{\alpha=x, y, z} \sum_{m=1}^{M_{i}} \sum_{n=1}^{N_{i}} \hat{\alpha} c_{i m n}^{\alpha} f_{i m n}(\bar{r})
$$

where $\alpha=x, y, z$ and $c_{i m n}^{\alpha}$ and $c_{j k l}^{s}$ are the unknown expansion coefficients.

Thus, the basic functions are given by the following

$$
\begin{aligned}
& f_{i m n}^{x}\left(x^{\prime}\right)=e^{j k_{x}^{i m n}\left(x^{\prime}-x_{o}^{i}\right)}, \quad f_{i m n}^{y}\left(y^{\prime}\right)=e^{j k_{y}^{i m n}\left(y^{\prime}-y_{o}^{i}\right)}, \quad f_{i m n}^{z}\left(z^{\prime}\right)=e^{j k_{z}^{i m n}\left(z^{\prime}-z_{o}^{i}\right)} \\
& f_{j k l}^{x}(x)=e^{j k_{x}^{j k l}\left(x-x_{o}^{i}\right)}, \quad f_{j k l}^{y}(y)=e^{j k_{y}^{j k l}\left(y-y_{o}^{i}\right)}, \quad f_{j k l}^{z}(z)=e^{j k_{z}^{j k l}\left(z-z_{o}^{i}\right)}
\end{aligned}
$$

Then Eq. (25) becomes

$$
\bar{e}_{i}\left(\bar{r}^{\prime}\right)=\sum_{m=1}^{M_{i}} \sum_{n=1}^{N_{i}}\left(\sum_{\alpha=x, y, z} \hat{\alpha}\right) c_{i m n}^{\alpha} e^{j k_{x}^{i m n}\left(x^{\prime}-x_{o}^{i}\right)} \cdot e^{j k_{y}^{i m n}\left(y^{\prime}-y_{o}^{i}\right)} \cdot e^{j k_{z}^{i m n}\left(z^{\prime}-z_{o}^{i}\right)}
$$


and after the implementation of the Galerkin technique the integral equations of the unknown electric field as stated in cases A, B and C are converted to the following respectively.

For Case A

$$
\begin{aligned}
\iiint_{V_{j}}(\overline{\overline{1}}+ & \left.\frac{k_{V}^{2}}{k_{o}^{2}} \delta \varepsilon_{r_{j}} \hat{z} \hat{z}\right) \sum_{m=1}^{M_{j}} \sum_{n=1}^{N_{j}}\left(\hat{s} \sum_{s=x, y, z} c_{j m n}^{s} \hat{s}\right) \\
\times & E_{o} e^{j k_{x}^{j k l}\left(x-x_{o}^{j}\right)} e^{j k_{y}^{j k l}\left(y-y_{o}^{j}\right)} e^{j k_{z}^{j k l}\left(z-z_{o}^{j}\right)} e^{j k_{x}^{j m n}\left(x-x_{o}^{j}\right)} e^{j k_{y}^{j m n}\left(y-y_{o}^{j}\right)} e^{j k_{z}^{j m n}\left(z-z_{o}^{j}\right)} \mathrm{d} x \mathrm{~d} y \mathrm{~d} z \\
= & \iiint_{V j} \iiint_{\text {everyyolume }} \hat{s} E_{o} e^{j k_{x}^{j k l}\left(x-x_{o}^{j}\right)} e^{j k_{y}^{j k l}\left(y-y_{o}^{j}\right)} e^{j k_{z}^{j k l}\left(z-z_{o}^{j}\right)}\left[\frac{k_{o}^{2}}{2 \pi} \sum_{i=1}^{N} \delta \varepsilon_{r_{i}} \bar{G}\left(\bar{r}, \bar{r}^{\prime}\right)\right. \\
& \times \sum_{m=1}^{M_{i}} \sum_{n=1}^{N_{i}}\left(\sum_{\alpha=x, y, z} \hat{\alpha} c_{i m n}^{\alpha}\right) e^{j k_{x}^{i m n}\left(x^{\prime}-x_{o}^{i}\right)} e^{j k_{y}^{i m n}\left(y^{\prime}-y_{o}^{i}\right)} e^{j k_{z}^{i m n}\left(z^{\prime}-z_{o}^{i}\right)} \\
& +\bar{G}\left(\bar{r}, \bar{r}^{\prime}\right)\left(\hat{y} J_{o} \cos \left(\frac{\pi x}{\alpha^{\prime}}\right) e^{-j \beta y}+\hat{y} R J_{o} \cos \left(\frac{\pi x}{\alpha^{\prime}}\right) e^{j \beta y}\right) \\
& \left.+\bar{G}\left(\bar{r}, \bar{r}^{\prime}\right) \hat{y} T J_{o} \cos \left(\frac{\pi x}{\alpha^{\prime}}\right) e^{-j \beta y}\right] \mathrm{d} x^{\prime} \mathrm{d} y^{\prime} \mathrm{d} z^{\prime} \mathrm{d} x \mathrm{~d} y \mathrm{~d} z
\end{aligned}
$$

For Case B

$$
\begin{aligned}
0=\iiint_{V_{W G 1}^{\prime \prime}} \hat{y} E_{o} \cos \left(\frac{\pi x}{\alpha^{\prime}}\right) e^{j \beta y} \mathrm{~d} x \mathrm{~d} y \mathrm{~d} z \iiint_{\text {everyvolume }}\left[\frac{k_{o}^{2}}{2 \pi} \sum_{i=1}^{N} \delta \varepsilon_{r_{i}} \bar{G}\left(\bar{r}, \bar{r}^{\prime}\right)\right. \\
\quad \times \sum_{m=1}^{M_{i}} \sum_{n=1}^{N_{i}}\left(\sum_{\alpha=x, y, z} \hat{\alpha}\right) c_{i m n}^{\alpha} e^{j k_{x}^{i m n}\left(x^{\prime}-x_{o}^{i}\right)} e^{j k_{y}^{i m n}\left(y^{\prime}-y_{o}^{i}\right)} e^{j k_{z}^{i m n}\left(z^{\prime}-z_{o}^{i}\right)} \\
+\bar{G}\left(\bar{r}, \bar{r}^{\prime}\right)\left(\hat{y} J_{o} \cos \left(\frac{\pi x}{\alpha^{\prime}}\right) e^{-j \beta y}+\hat{y} R J_{o} \cos \left(\frac{\pi x}{\alpha^{\prime}}\right) e^{j \beta y}\right) \\
\left.+\bar{G}\left(\bar{r}, \bar{r}^{\prime}\right) \hat{y} T J_{o} \cos \left(\frac{\pi x}{\alpha^{\prime}}\right) e^{-j \beta y}\right] \mathrm{d} x^{\prime} \mathrm{d} y^{\prime} \mathrm{d} z^{\prime}
\end{aligned}
$$

For Case C

$$
\begin{aligned}
0=\iiint_{V_{W G 2}^{\prime \prime}} \hat{y} E_{o} \cos \left(\frac{\pi x}{\alpha^{\prime}}\right) e^{-j \beta y} \mathrm{~d} x \mathrm{~d} y \mathrm{~d} z \iiint_{\text {everyvolume }}\left[\frac{k_{o}^{2}}{2 \pi} \sum_{i=1}^{N} \delta \varepsilon_{r_{i}} \bar{G}\left(\bar{r}, \bar{r}^{\prime}\right)\right. \\
\quad \times \sum_{m=1}^{M_{i}} \sum_{n=1}^{N_{i}}\left(\sum_{\alpha=x, y, z} \hat{\alpha}\right) c_{i m n}^{\alpha} e^{j k_{x}^{i m n}\left(x^{\prime}-x_{o}^{i}\right)} e^{j k_{y}^{i m n}\left(y^{\prime}-y_{o}^{i}\right)} e^{j k_{z}^{i m n}\left(z^{\prime}-z_{o}^{i}\right)} \\
+\bar{G}\left(\bar{r}, \bar{r}^{\prime}\right)\left(\hat{y} J_{o} \cos \left(\frac{\pi x}{\alpha^{\prime}}\right) e^{-j \beta y}+\hat{y} R J_{o} \cos \left(\frac{\pi x}{\alpha^{\prime}}\right) e^{j \beta y}\right) \\
\left.+\bar{G}\left(\bar{r}, \bar{r}^{\prime}\right) \hat{y} T J_{o} \cos \left(\frac{\pi x}{\alpha^{\prime}}\right) e^{-j \beta y}\right] \mathrm{d} x^{\prime} \mathrm{d} y^{\prime} \mathrm{d} z^{\prime}
\end{aligned}
$$


Reference to the above analysis the initial system of integral equations has been converted to an equivalent algebraic one whose general formulation [9] is presented by the following matrix

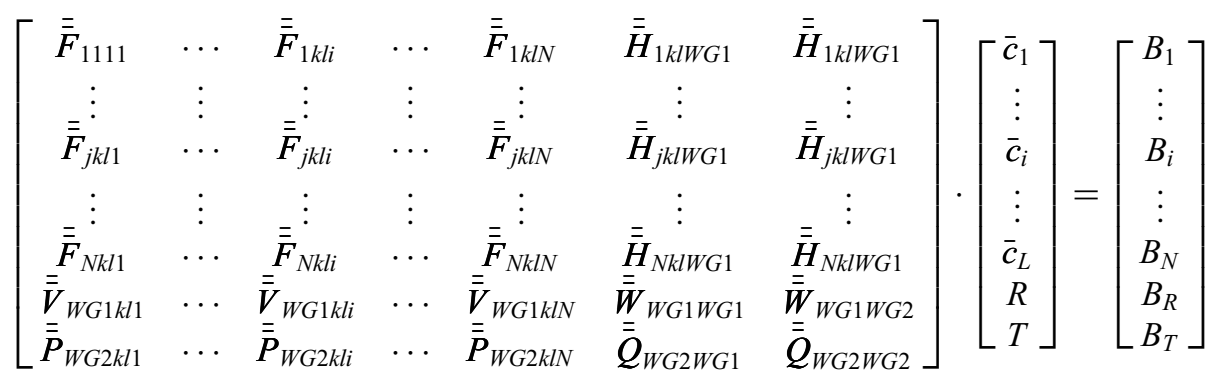

where a submatrix $\mathbf{F}_{j i}$ is being formulated by the expression sGa or in other words by

$$
\overline{\bar{F}}_{j i}=\left[\begin{array}{ccc}
\overline{\bar{F}}_{j i}^{x x} & \overline{\bar{F}}_{j i}^{x y} & \overline{\bar{F}}_{j i}^{x z} \\
\overline{\bar{F}}_{j i} & \overline{\bar{F}}_{j i} & \overline{\bar{F}}_{j i}^{y z} \\
\overline{\bar{F}}_{j i} & \overline{\bar{F}}_{j i}^{z y} & \overline{\bar{F}}_{j i}^{z z}
\end{array}\right]
$$

and $\bar{c}_{i}$ the unknown expansion coefficients, $R$ and $T$ the reflection and transfer coefficients respectively. The dimension of the derived matrix is $\left(N_{\text {tot }}+2\right) \times\left(N_{\text {tot }}+2\right)$ where

$$
N_{\text {tot }}=3 x\left(\left(N_{1} x M_{1}\right)+\left(N_{2} x M_{2}\right)+\cdots+\left(N_{N} x M_{N}\right)\right)=3 x \sum_{i=1}^{N}\left(N_{i} x M_{i}\right)
$$

\section{ANALYTICAL SOLUTION}

Reference to the three cases stated above, each equation contains a number of integrals that allow of analytical solution, while others and particularly those including the Green functions' expression can only be solved by numerical computation. In the following, case A is being considered as an example, in order to show the amount of operations involved and the way the final system was treated in terms of the analytical and numerical computation [9].

Rearranging terms in case A equations becomes

$$
\begin{aligned}
\int_{x_{1}^{j}}^{x_{2}^{j}} \int_{y_{1}^{j}}^{y_{2}^{j}} \int_{z_{1}^{j}}^{z_{2}^{j}} \int_{x_{1}^{i}}^{x_{2}^{i}} \int_{y_{1}^{i}}^{y_{2}^{i}} \int_{z_{1}^{i}}^{z_{2}^{i}} k_{o}^{2} E_{o}^{2} \sum_{i=1}^{N} \delta \varepsilon_{r_{i}} \sum_{m=1}^{M_{i}} \sum_{n=1}^{N_{i}} \sum_{\alpha=x, y, z} \hat{s} \overline{\bar{G}}\left(\bar{r}, \bar{r}^{\prime}\right) \hat{\alpha} c_{i m n}^{\alpha} E_{o} e^{j k_{x}^{i m n}\left(x^{\prime}-x_{o}^{i}\right)} e^{j k_{y}^{i m n}\left(y^{\prime}-y_{o}^{i}\right)} \\
\quad \times e^{j k_{z}^{i m n}\left(z^{\prime}-z_{o}^{i}\right)} e^{j k_{x}^{j k l\left(x-x_{o}^{j}\right)}} e^{j k_{y}^{j k\left(y-y_{o}^{j}\right)}} e^{j k_{z}^{j k l\left(z-z_{o}^{j}\right)}} \mathrm{d} x^{\prime} \mathrm{d} y^{\prime} \mathrm{d} z^{\prime} \mathrm{d} x \mathrm{~d} y \mathrm{~d} z \\
-\int_{x_{1}^{j}}^{x_{2}^{j}} \int_{y_{1}^{j}}^{y_{2}^{j}} \int_{z_{1}^{j}}^{z_{2}^{j}} \sum_{m=1}^{M_{j}} \sum_{n=1}^{N_{j}}\left(\hat{s} \sum_{s=x, y, z} c_{j m n}^{s} \hat{s}\right) E_{o} e^{j k_{x}^{j m n}\left(x-x_{o}^{j}\right)} e^{j k_{x}^{j k l}\left(x-x_{o}^{j}\right)} e^{j k_{y}^{j m n}\left(y-y_{o}^{j}\right)} e^{j k_{y}^{j k l}\left(y-y_{o}^{j}\right)}
\end{aligned}
$$




$$
\begin{aligned}
& \times e^{j k_{z}^{j m n}\left(z-z_{o}^{j}\right)} e^{j k_{z}^{j k l}\left(z-z_{o}^{j}\right)}+\int_{x_{1}^{j}}^{x_{2}^{j}} \int_{y_{1}^{j}}^{y_{2}^{j}} \int_{z_{1}^{j}}^{z_{2}^{j}} \int_{x_{K 11}}^{x_{K 12}} \int_{-\infty}^{y_{K 12}} \int_{z_{K 11}}^{z_{K 12}} k_{o}^{2} \delta \varepsilon_{r_{K 1}} \hat{s} \overline{\bar{G}}\left(\bar{r}, \bar{r}^{\prime}\right) \hat{y} R E_{o}^{2} \cos \left(\frac{\pi x^{\prime}}{\alpha^{\prime}}\right) \\
& \times e^{j \beta y^{\prime}} e^{j k_{x}^{j k l}\left(x-x_{o}^{j}\right)} e^{j k_{y}^{j k l}\left(y-y_{o}^{j}\right)} e^{j k_{z}^{j k l}\left(z-z_{o}^{j}\right)} \mathrm{d} x^{\prime} \mathrm{d} y^{\prime} \mathrm{d} z^{\prime} \mathrm{d} x \mathrm{~d} y \mathrm{~d} z \\
& +\int_{x_{1}^{j}}^{x_{2}^{j}} \int_{y_{1}^{j}}^{y_{2}^{j}} \int_{z_{1}^{j}}^{z_{2}^{j}} \int_{x_{K 21}}^{x_{K 22}} \int_{y_{K 21}}^{+\infty} \int_{z_{K 21}}^{z_{K 22}} k_{o}^{2} \delta \varepsilon_{r_{K 2}} \hat{s} \overline{\bar{G}}\left(\bar{r}, \bar{r}^{\prime}\right) \hat{y} T E_{o}^{2} \cos \left(\frac{\pi x^{\prime}}{\alpha^{\prime}}\right) e^{-j \beta y^{\prime}} e^{j k_{x}^{j k l}\left(x-x_{o}^{j}\right)} e^{j k_{y}^{j k l}\left(y-y_{o}^{j}\right)} \\
& \times e^{j k_{z}^{j k l}\left(z-z_{o}^{j}\right)} \mathrm{d} x^{\prime} \mathrm{d} y^{\prime} \mathrm{d} z^{\prime} \mathrm{d} x \mathrm{~d} y \mathrm{~d} z=-\int_{x_{1}^{j}}^{x_{2}^{j}} \int_{y_{1}^{j}}^{y_{2}^{j}} \int_{z_{1}^{j}}^{z_{2}^{j}} \int_{x_{K 11}}^{x_{K 12}} \int_{-\infty}^{y_{K 12}} \int_{z_{K 11}}^{z_{K 12}} k_{o}^{2} \delta \varepsilon_{r_{K 1}} \hat{s} \overline{\bar{G}}(\bar{r}, \bar{r}) \hat{y} E_{o}^{2} \\
& \times \cos \left(\frac{\pi x^{\prime}}{\alpha^{\prime}}\right) e^{-j \beta y^{\prime}} e^{j k_{x}^{j k l}\left(x-x_{o}^{j}\right)} e^{j k_{y}^{j k l}\left(y-y_{o}^{j}\right)} e^{j k_{z}^{j k l}\left(z-z_{o}^{j}\right)} \mathrm{d} x^{\prime} \mathrm{d} y^{\prime} \mathrm{d} z^{\prime} \mathrm{d} x \mathrm{~d} y \mathrm{~d} z
\end{aligned}
$$

Considering the first term of the left hand side

$$
\begin{aligned}
\iiint_{V_{j}} & \iint_{V_{i}} \int_{o} k_{o}^{2} E_{o} \sum_{i=1}^{N} \delta \varepsilon_{r_{i}} \sum_{m=1}^{M_{i}} \sum_{n=1}^{N_{i}} \sum_{\alpha=x, y, z}\left(\hat{\bar{s}} \overline{\bar{G}}\left(\bar{r}, \bar{r}^{\prime}\right) \hat{\alpha}\right) c_{i m n}^{\alpha} e^{j k_{x}^{i m n}\left(x^{\prime}-x_{o}^{i}\right)} e^{j k_{y}^{i m n}\left(y^{\prime}-y_{o}^{j}\right)} e^{j k_{z}^{i m n}\left(z^{\prime}-z_{o}^{i}\right)} \\
& \times e^{j k_{x}^{j k l}\left(x-x_{o}^{j}\right)} e^{j k_{y}^{j k l}\left(y-y_{o}^{j}\right)} e^{j k_{z}^{j k l}\left(z-z_{o}^{j}\right)} \mathrm{d} x \mathrm{~d} y \mathrm{~d} z \mathrm{~d} x^{\prime} \mathrm{d} y^{\prime} \mathrm{d} z^{\prime}
\end{aligned}
$$

which corresponds to the assumption of field sources being the cells inside the circuit after implementing the Galerkin technique.

This expression can be written in the following way

$$
\begin{gathered}
E_{o} \frac{k_{o}^{2}}{(2 \pi)^{2}} \sum_{i=1}^{N} \delta \varepsilon_{r_{i}} \sum_{m=1}^{M_{i}} \sum_{n=1}^{N_{i}} \sum_{\alpha=x, y, z} \int_{x_{1}^{j}}^{x_{2}^{j}} \int_{x_{1}^{i}}^{x_{2}^{i}} e^{j k_{x}^{j k l}\left(x-x_{o}^{j}\right)} e^{j k_{x}^{i m n}\left(x^{\prime}-x_{o}^{i}\right)} \int_{y_{1}^{j}}^{y_{2}^{j}} \int_{y_{1}^{i}}^{y_{2}^{i}} e^{j k_{y}^{j k l}\left(y-y_{o}^{j}\right)} e^{j k_{y}^{i m n}\left(y^{\prime}-y_{o}^{i}\right)} \\
\times \int_{z_{1}^{j}}^{z_{2}^{j}} \int_{z_{1}^{i}}^{z_{2}^{i}} \int_{-\infty}^{+\infty} \int_{-\infty}^{+\infty}(\hat{s} \overline{\bar{g}} \hat{\alpha}) e^{j k_{z}^{j k l}\left(z-z_{o}^{j}\right)} e^{j k_{z}^{i m n}\left(z^{\prime}-z_{o}^{i}\right)} e^{j\left(k_{x}\left(x-x^{\prime}\right)+k_{y}\left(y-y^{\prime}\right)\right)} c_{i m n}^{\alpha} \mathrm{d} k_{x} \mathrm{~d} k_{y}
\end{gathered}
$$

where from a simple inspection it is obvious that the integrals corresponding to variables $x, y$ and $z$ can be solved analytically (with some assumptions taken into account for the latter). For convinience the above equation can be rewritten as

$$
E_{o} \frac{k_{o}^{2}}{(2 \pi)^{2}} \sum_{i=1}^{N} c_{i m n}^{\alpha} \delta \varepsilon_{r_{i}} \sum_{m=1}^{M_{i}} \sum_{n=1}^{N_{i}} \sum_{\alpha=x, y, z} \int_{-\infty}^{+\infty} \int_{-\infty}^{+\infty} I X_{N N} I Y_{N N} I Z_{N N}(\hat{s} \overline{\bar{g}} \hat{\alpha}) \mathrm{d} k_{x} \mathrm{~d} k_{y}
$$

where the correspondence is self-evident. Thus the $I X_{N N}, I Y_{N N}$ can be computed analytically easily. The analytical solution of the $I Z_{N N}$ term depends on the relative position of the $j$ cell (observation point) and $i$ cell (field sources) towards $z$-axis. Since dyadic Green function can be expressed in terms of

$$
\overline{\bar{g}}=\left\lfloor\overline{\bar{g}}_{1} e^{-j k_{z o}\left(z+z^{\prime}\right)}+\overline{\bar{g}}_{2} e^{j k_{z o}\left(z-z^{\prime}\right)} u\left(z^{\prime}-z\right)+\overline{\bar{g}}_{3} e^{-j k_{z o}\left(z-z^{\prime}\right)} u\left(z-z^{\prime}\right)\right\rfloor
$$

$\overline{\bar{g}}_{1}$ term being the secondary term while $\overline{\bar{g}}_{2}$ and $\overline{\bar{g}}_{3}$ terms refer to the primary one. 
Discrete cases should be examined reference to the step function used. For instance if cell $j$ lies above cell $i$ (as represented in Fig. 2) then

$$
u\left(z^{\prime}-z\right)=0 \quad u\left(z-z^{\prime}\right)=1 \quad z>z^{\prime}
$$

which results in

$$
I Z_{N N}^{\mathrm{pr}}=\int_{z_{1}^{j}}^{z_{2}^{j}} \int_{z_{1}^{i}}^{z_{2}^{i}} e^{-j k_{z o}\left(z-z^{\prime}\right)} e^{j k_{z}^{j k l}\left(z-z_{o}^{j}\right)} e^{j k k_{z}^{i m n}\left(z^{\prime}-z_{o}^{i}\right)} \mathrm{d} z \mathrm{~d} z^{\prime} \int_{-\infty}^{+\infty} \int_{-\infty}^{+\infty}\left(\hat{s} \overline{\bar{g}}_{3} \hat{\alpha}\right) \mathrm{d} k_{x} \mathrm{~d} k_{y}
$$

where the first integral term is being solved analytically. Similar results are obtained if cell $j$ lies beneath $i$-cell while in case where the cells repose the same projection in $z$ axis all terms are taken into account.

\section{Propagation Constant}

The propagation constant $\beta$ being complex quantity is given by

$$
\beta=\beta_{r}-j \beta_{i}
$$

where $\beta_{r}$ represents the phase rotation and $\beta_{i}$ represents the dielectric and ohmic loss (attenuation) in general [9]. It is also assumed that

$$
\beta=\beta_{r}-j \beta_{i} \cong \beta_{r}, \quad \beta_{i} \ll \beta_{r}
$$

that is, the imaginary part it is assumed relatively small in comparison to the real one allowing the presumption of a real propagation constant only for the incident wave. In other words, a justified approximation is taken into account assuming that the incident wave propagation constant is real since the incident wave is free of losses. In all circumstances the propagation constant is a datum to the analysis and its value is obtained through a way certified for its correctness. The numerical value of the propagation constant $\beta$ is obtained from the characteristics of the microstrip line as they are computed from the Computer Aided Design Software Libra Touchstone of HP-Eesof.

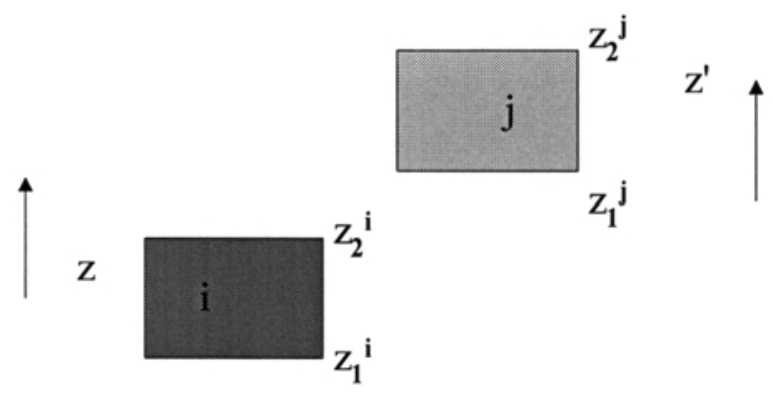

FIGURE 2 Relevant position of $i$ and $j$ cells 


\section{NUMERICAL SOLUTION - APPLICATIONS AND RESULTS}

From the procedure described above it is evident that the same algorithm is pursued in order to develop the final expressions and therefore to define the formulation of every element of the final system matrix. It is obvious that for more sophisticated circuits the system becomes even larger and more complicated. The numerical computation is applied for Green function's infinite integrals where a major issue concerning the convergence is encountered. Simpson method is being used for numerical integration which results to a very large computation time in CPU terms due to the fact that involves double infinite integrals. Regarding the matrix inversion several methods were implemented in order to ensure the correct results.

As it was mentioned earlier the Method of Moments - Galerkin technique assumes the field approximation with the superposition of plane waves. By that way, the more plane waves are used the better field approximation is being made resulting to even more complicated system. On the other hand, some of the formulated expressions involve functions that oscillate rapidly, which effects the retardation of the convergence. Therefore, the computation time becomes very large even for elementary applications of simple geometries with several plane waves superimposed.

This problem has been solved in a satisfactory way with the introduction of Parallel Processing - High Performance Computing techniques while Multiprocessing machines were used [13]. By that way a large number of the functions consisting the matrix elements is being computed simultaneously exploiting the CPU capabilities. Several results [9] that study different longitudinal discontinuity cases are derived which show the connection between the field coefficients inside the cells along with $R$ and $T$ coefficients. Specific results are presented in the following for the structure shown in Figure 3 where the analysis data are given below:

Frequency of operation: $50 \mathrm{GHz}$

\section{Substrate Characteristics}

1st layer's height: $d_{1}=22.7 \mu \mathrm{m}$

1st layer's dielectric constant: $\varepsilon_{r_{1}}=10$

1st layer's conductivity: $\sigma_{1}=0.00278$ 2nd layer's height: $d_{2}=17.1 \mu \mathrm{m}$

2nd layer's dielectric constant: $\varepsilon_{r_{2}}=12.85$

2nd layer's conductivity: $\sigma_{2}=0.00278$

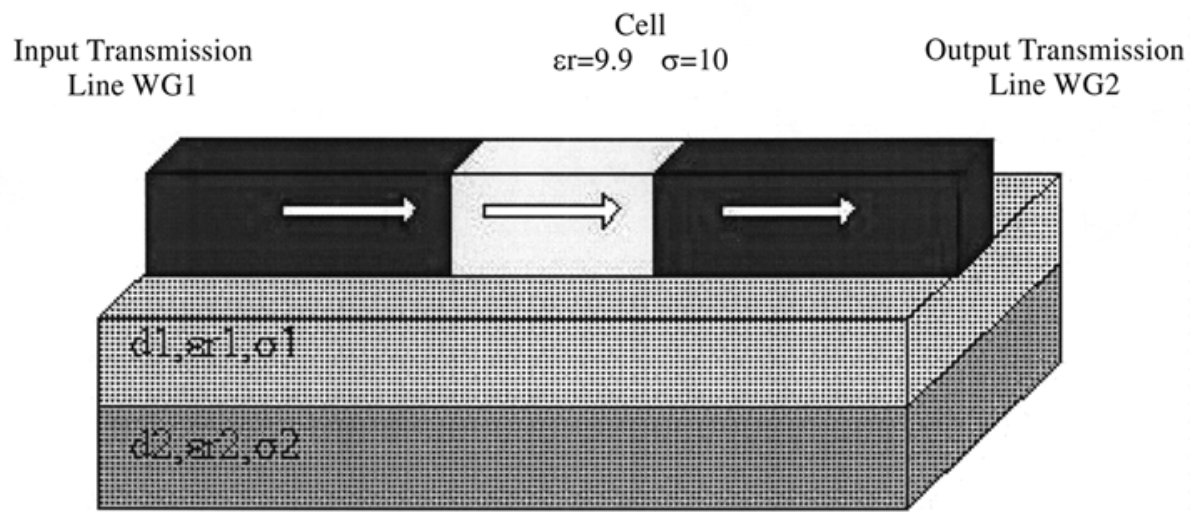

FIGURE 3 Application structure 
Complex propagation constant: $\beta=2755.78-j 18.193$

Dimensions of microstrip lines and cells:

Input microstrip line dimensions: $l_{x}=30 \mu \mathrm{m}, l_{y}=0.5 \mathrm{~m}, l_{z}=5 \mu \mathrm{m}$

Output microstrip line dimensions: $l_{x}=30 \mu \mathrm{m}, l_{y}=0.5 \mathrm{~m}, l_{z}=5 \mu \mathrm{m}$

Dielectric cell dimensions: $l_{x}=30 \mu \mathrm{m}, l_{y}=953 \mu \mathrm{m}, l_{z}=5 \mu \mathrm{m}$

\section{Electrical Characteristics of the Cell}

Dielectric constant: $\varepsilon_{r}=9.9$

Conductivity: $\sigma=10$

The arrow in Figure 3 represents the direction of the assumed one plane wave in the geometry under consideration (weak approximation). The system matrix involves 25 elements while the numerical results are given below

$$
\begin{aligned}
c_{i m n}^{x} & =-1.68 \times 10^{-11}+j 1.88 \times 10^{-11} \\
c_{i m n}^{y} & =1.98 \times 10^{-3}+j 3.53 \times 10^{-4} \\
c_{i m n}^{z} & =-1.16 \times 10^{-4}+j 6.89 \times 10^{-5} \\
R & =0.768-j 0.553 \\
T & =-5.028 \times 10^{-2}-j 3.61 \times 10^{-2}
\end{aligned}
$$

From the above results it is noticed that despite the weak approximation, the $y$-component of the electric field as expressed by the coefficients values is the dominant one since the wave propagates along the $y$-axis. A leakage occurs along $z$-axis, which is predictable due to the capacitance between the transmission lines and the dielectric cell as well as between the transmission lines, the ground plane and the dielectric substrate layers. Finally, the $x$-component is extremely small in comparison to the others since no significant filed is expected along $x$-axis.

As a next step varying some of the structure's characteristics, several results are derived. For instance, when the cell's conductivity becomes larger $(\sigma=500)$ the results vary as follows

$$
\begin{aligned}
c_{i m n}^{x} & =2.94 \times 10^{-14}+j 4.67 \times 10^{-14} \\
c_{i m n}^{y} & =2.06 \times 10^{-6}-j 9.65 \times 10^{-6} \\
c_{i m n}^{z} & =2.51 \times 10^{-7}-j 1.265 \times 10^{-7} \\
R & =0.865-j 0.597 \\
T & =-0.146-j 4.67 \times 10^{-2}
\end{aligned}
$$

From the above results it is shown that the relations between the field components are the same: the $y$-component of the electric field is still the larger one while the others are less. For all cases, the coefficients in general, become less in value, at least two orders of magnitude, in comparison to the previous ones, due to the fact that the cell losses and therefore the field attenuation is now increased. Similar conclusions are derived for the $R$ and $T$ coefficients, where the latter is almost 3.5 times larger. That becomes due to the fact that in the second case the electric field faces less resistance propagating through the cell to the output transmission line. 


\section{NON-LINEAR ANALYSIS}

The method described in the previous sections consists of a Generalized Three Dimensional full wave dynamic solution to analyze MMIC structures. Both the method described and the results presented refer only to cells with linear characteristics in terms of dielectric permittivity and conductivity. Despite this fact the method described is a general one and with specific modifications can be applied for non-linear cell mediums without changing vast the initial assumptions. The analysis for the non-linear case is presented in the following.

A harmonic analysis technique will be introduced in order to solve the non-linear properties of the circuit. The new issue introduced is the aspect of non-linearity that is confined in a finite orthogonal parallelopiped region between linear cells. So everything that has been described in Figure 1 is still valid with the addition that one of the linear blocks can be viewed as the perturbating non-linear area.

Reference to an excitation consisting of two plane waves at the discrete frequencies $\omega_{1}, \omega_{2}$ the main goal will be to determine the unknown electric field oscillating at the intermediate frequency (IF) $\omega=\omega_{1}-\omega_{2}\left(\omega_{1}>\omega_{2}\right)$. In general the unknown electric field inside the $3 \mathrm{D}$ non-linear region could be described with the use of an integral equation as follows:

$$
\underline{E}(\underline{r}, \omega)=\underline{E}_{o}(\underline{r}, \omega)+\frac{\omega^{2}}{c^{2}} \iiint_{V} \underline{\bar{G}}\left(\underline{r}, \underline{r}^{\prime}, \omega\right) \stackrel{\circ}{X}\left(\underline{r}^{\prime}, \omega\right) E\left(\underline{r}^{\prime}, \omega\right) \mathrm{d} V\left(r^{\prime}\right)
$$

Where $\underline{E}_{o}(\underline{r}, \omega)$ is the incident electric field and $X\left(\underline{r}^{\prime}, \omega\right)=\left(\varepsilon_{r}\left(\underline{r}^{\prime}, \omega\right)-1\right)$ is the complex suspectibility while $\underline{\bar{G}}\left(\underline{r}, \underline{r}^{\prime}, \omega\right)$ is the Green's function used [7]. The complex suspectibility is also connected to the unknown electric field and the charge surface density through the following relation:

$$
\underline{Q}(\underline{r}, \omega)=\stackrel{\circ}{X}(\underline{r}, \omega) E(\underline{r}, \omega)
$$

Assuming a weak non-linearity condition the Wiener-Voltera Method could be applied and the surface charge density could be expressed with the use of a voltera series as:

$$
\begin{aligned}
Q_{I}(\underline{r}, t)= & \int_{-\infty}^{+\infty} \mathrm{d} \xi_{1} x_{1}\left(\underline{r}, t-\xi_{1}\right) \\
& \times\left\{\sum_{n=-N}^{N} \sum_{m=-M}^{M} \frac{1}{2}\left[e_{i, n m}(\underline{r}) e^{j\left(n \omega_{1}+m \omega_{2}\right) \xi_{1}}+e_{i, n m}^{*}(\underline{r}) e^{-j\left(n \omega_{1}+m \omega_{2}\right) \xi_{1}}\right]\right\} \\
& +\int_{-\infty}^{+\infty} \mathrm{d} \xi_{1} \int_{-\infty}^{+\infty} \mathrm{d} \xi_{2} x_{2}\left(\underline{r}, t-\xi_{1}, t-\xi_{2}\right) \frac{1}{2^{2}} \\
& \times\left\{\sum_{n_{1}}^{N_{1}} \sum_{m_{1}}^{M_{1}}\left[e_{i, n_{1} m_{1}}(\underline{r}) e^{j\left(n_{1} \omega_{1}+m_{1} \omega_{2}\right) \xi_{1}}+e_{i, n_{1} m_{1}}^{*}(\underline{r}) e^{-j\left(n_{1} \omega_{1}+m_{1} \omega_{2}\right) \xi_{1}}\right]\right. \\
& \left.\cdot \sum_{n_{2}}^{N_{2}} \sum_{m_{2}}^{M_{2}}\left[e_{i, n_{2} m_{2}}(\underline{r}) e^{j\left(n_{2} \omega_{1}+m_{2} \omega_{2}\right) \xi_{2}}+e_{i, n_{2} m_{2}}^{*}(\underline{r}) e^{-j\left(n_{2} \omega_{1}+m_{2} \omega_{2}\right) \xi_{2}}\right]+\cdots+\right\}
\end{aligned}
$$

where $(i=x, y, z)$ and $\omega_{n m}=n \omega_{1}+m \omega_{2}$. The unknown $x_{1}, x_{2}, x_{3}, \ldots, x_{n}$ functions are connected to the permittivity $\varepsilon_{r}$. In the previous equation the unknown electric field $E_{i}$, has been expressed as a sum of plane waves. The values of $N, M$ will be set according to the number of harmonics needed for the most accurate expression of the unknown electric field. 
Equation (43) in the frequency domain becomes:

$$
\begin{aligned}
\tilde{Q}_{i}(\underline{r}, \omega)=2 \pi\{ & \frac{1}{2} \sum_{n}^{N} \sum_{m}^{M}\left[\delta\left(\omega-\omega_{n m}\right) e_{i, n m}(\underline{r}) X_{1}\left(\underline{r}, \omega_{n m}\right)+\delta\left(\omega+\omega_{n m}\right) e_{i, n m}^{*}(\underline{r}) X_{1}\left(\underline{r},-\omega_{n m}\right)\right] \\
& +\frac{1}{2^{2}} \sum_{n_{1}}^{N_{1}} \sum_{m_{1}}^{M_{1}} \sum_{n_{2}}^{N_{2}} \sum_{m_{2}}^{M_{2}}\left[\left\{e_{n_{1} m_{1}} e_{n_{2} m_{2}}\right\} \delta\left(\omega-\left(\omega_{n_{1} m_{1}}+\omega_{n_{2} m_{2}}\right)\right) X_{2}\left(\underline{r}, \omega_{n_{1} m_{1}}, \omega_{n_{2} m_{2}}\right)\right. \\
& +\left\{e_{n_{1} m_{1}} e_{n_{2} m_{2}}^{*}\right\} \delta\left(\omega-\left(\omega_{n_{1} m_{1}}-\omega_{n_{2} m_{2}}\right)\right) X_{2}\left(\underline{r}, \omega_{n_{1} m_{1}},-\omega_{n_{2} m_{2}}\right)\left\{e_{n_{1} m_{1}}^{*} e_{n_{2} m_{2}}\right\} \\
& \times \delta\left(\omega-\left(-\omega_{n_{1} m_{1}}+\omega_{n_{2} m_{2}}\right)\right) X_{2}\left(\underline{r},-\omega_{n_{1} m_{1}}, \omega_{n_{2} m_{2}}\right)+\left\{e_{n_{1} m_{1}}^{*} e_{n_{2} m_{2}}^{*}\right\} \\
& \left.\left.\times \delta\left(\omega-\left(-\omega_{n_{1} m_{1}}-\omega_{n_{2} m_{2}}\right)\right) X_{2}\left(\underline{r},-\omega_{n_{1} m_{1}},-\omega_{n_{2} m_{2}}\right)+\frac{1}{2^{3}}+\cdots\right]\right\}
\end{aligned}
$$

where

$$
\begin{aligned}
\underline{e}_{n_{1} m_{1}}(\underline{r}) \cdot \underline{e}_{n_{2} m_{2}}(\underline{r}) & =e_{i, n_{1} m_{1}} \cdot(\underline{r}) e_{i, n_{2} m_{2}}(\underline{r})=e_{n_{1} m_{1}}^{x} e_{n_{2} m_{2}}^{x} \hat{x}+e_{n_{1} m_{1}}^{y} e_{n_{2} m_{2}}^{y} \hat{y}+e_{n_{1} m_{1}}^{z} e_{n_{2} m_{2}}^{z} \hat{z} \\
& \equiv\left\{e_{n_{1} m_{1}} e_{n_{2} m_{2}}\right\}
\end{aligned}
$$

Equation (44) is the final form of the charge surface density in the frequency field. Transferring the unknown electric field and the incident one in the frequency domain and using Eq. (42) and Eq. (41) becomes:

$$
\begin{aligned}
& \pi \sum_{n=-N}^{N} \sum_{\substack{m=-M \\
n=m \neq 0}}^{M}\left[e_{i, n m}(\underline{r}) \delta\left(\omega-\omega_{n m}\right)+e_{i, n m}^{*}(\underline{r}) \delta\left(\omega+\omega_{n m}\right)\right] \\
& \quad=\pi\left[e_{0,1}^{y}(\underline{r}) \delta\left(\omega-\omega_{1}\right)+e_{0,2}^{y}(\underline{r}) \delta\left(\omega-\omega_{2}\right)+c c\right]+\frac{\omega^{2}}{c^{2}} \iiint_{V} \underline{\underline{G}}\left(\underline{r}, \underline{r}^{\prime}, \omega\right) \underline{\tilde{Q}}\left(\underline{r}^{\prime}, \omega\right) \mathrm{d} V\left(\underline{r}^{\prime}\right)
\end{aligned}
$$

Where $c c$ is the complex conjugate and $\tilde{Q}\left(r^{\prime}, \omega\right)$ is given by (44). The previous equation is valid for each one of the discrete $\omega_{1}, \omega_{2}, \ldots, \omega_{n m}$ frequencies. Since the assumption of a non-linear region has been made, the unknown electric field can be expressed in general form as follows:

$$
\underline{e}_{n m}(\underline{r})=\underline{e}_{n m}^{l n}(\underline{r})+\delta e_{n m}^{n l}(\underline{r})
$$

Equation (47) denotes that the unknown electric field is the sum of two terms. The first term is the contribution of the linear cells to the total solution and the second the contribution of the non-linear region. The first term has been determined from the linear analysis so our main interest is the second term $\delta e_{n m}^{n l}(\underline{r})$. Solving Eq. (46) for the intermediate frequency $\omega=\omega_{1}-\omega_{2}$, which is the output frequency of the circuit, it is derived:

$$
\begin{aligned}
e_{i, 1-1}(\underline{r}) & +e_{i,-11}^{*}(\underline{r}) \\
= & 0+\frac{\left(\omega_{1}-\omega_{2}\right)^{2}}{c^{2}} \iiint_{V} \mathrm{~d} V\left(\underline{r^{\prime}}\right) \underline{\bar{G}}\left(\underline{r}, \underline{r}^{\prime}, \omega_{1}-\omega_{2}\right)\left[\left(e_{i, 1-1}\left(\underline{r}^{\prime}\right)+e_{i,-11}\left(\underline{r}^{\prime}\right)\right) X_{1}\left(\underline{r}^{\prime}, \omega_{1}-\omega_{2}\right)\right] \\
& +\frac{\left(\omega_{1}-\omega_{2}\right)^{2}}{2 c^{2}} \iiint \int \mathrm{d} V\left(\underline{r}^{\prime}\right) \underline{\bar{G}}\left(\underline{r}, \underline{r}^{\prime}, \omega_{1}-\omega_{2}\right)\left[4 \{ e _ { 1 0 } e _ { 0 1 } ^ { * } \} \left(X_{2}\left(\underline{r}^{\prime}, \omega_{1},-\omega_{2}\right)\right.\right. \\
& \left.\left.+X_{2}\left(\underline{r}^{\prime},-\omega_{2}, \omega_{1}\right)\right)\right]
\end{aligned}
$$


In this equation the excitation term does not exist because there is no incident wave at $\omega=\omega_{1}-\omega_{2}$. Returning to Eq. (47) for the frequency $\omega=\omega_{1}-\omega_{2}$ stands:

$$
\underline{e}_{n m}(\underline{r})=\underline{e}_{n m}^{l n}(\underline{r})+\delta e_{n m}^{n l}(\underline{r}) \Rightarrow \underline{e}_{1-1}(\underline{r})=\underline{e}_{1-1}^{l n}(\underline{r})+\delta e_{1-1}^{n l}(\underline{r})
$$

From the previous equation we may observe that $\underline{e}_{1-1}^{l n}(\underline{r})=0$ since there is no excitation at $\omega=\omega_{1}-\omega_{2}$ frequency. So the non-linear term becomes:

$$
\begin{aligned}
\delta e_{1-1}^{n l}(\underline{r})= & \frac{2}{c^{2}} \iiint_{V} \mathrm{~d} V\left(\underline{r}^{\prime}\right)\left(\omega_{1}-\omega_{2}\right)^{2} \underline{\bar{G}}\left(\underline{r}, \underline{r}^{\prime}, \omega_{1}-\omega_{2}\right)\left[\{ e _ { 1 0 } e _ { 0 1 } ^ { * } \} \left(X_{2}\left(\underline{r}^{\prime}, \omega_{1},-\omega_{2}\right)\right.\right. \\
& \left.\left.+X_{2}\left(\underline{r}^{\prime},-\omega_{2}, \omega_{1}\right)\right)\right]
\end{aligned}
$$

Equation (50) is an approximation of the accurate solution since we have made the assumption that the non-linear term $\delta e_{1-1}^{n l}(\underline{r})$ does not appear in the linear part of Eq. (48). Equation (50) is the final form of the non-linear term, which represents the unknown electric field inside the non-linear region. It can be further simplified if we take into consideration the observation made from the numerical results of the linear analysis that the $\hat{y}$ component of the electric field is the dominant one. So Eq. (50) becomes:

$$
\begin{aligned}
\delta e_{1-1}^{n l}(\underline{r}) \hat{y}= & \frac{2}{c^{2}} \iiint_{V} \mathrm{~d} V\left(\underline{r}^{\prime}\right)\left(\omega_{1}-\omega_{2}\right)^{2} \hat{y} \underline{G}\left(\underline{r}, \underline{r}^{\prime}, \omega_{1}-\omega_{2}\right) \hat{y}\left[( e _ { 1 0 } ^ { y } e _ { 0 1 } ^ { y * } ) \left(X_{2}\left(\underline{r}^{\prime}, \omega_{1},-\omega_{2}\right)\right.\right. \\
& \left.\left.+X_{2}\left(\underline{r}^{\prime},-\omega_{2}, \omega_{1}\right)\right)\right]
\end{aligned}
$$

Furthermore since $\omega_{1} \approx \omega_{2} \approx 50 \mathrm{GHz}$ (this is a typical value for the operational frequency from the linear analysis numerical results) it can be assumed that $\omega_{1}-\omega_{2} \stackrel{\omega_{1} \approx \omega_{2}}{\longrightarrow} 0$. Thus calculating the non-linear term results in calculating the following limit:

$$
\delta e_{1-1}^{n l}(\underline{r}) \hat{y}=\lim _{\Omega \rightarrow 0} \frac{2}{c^{2}} \iiint_{V} \mathrm{~d} V\left(\underline{r}^{\prime}\right) \Omega^{2} \hat{y} \underline{\bar{G}}\left(\underline{r}, \underline{r}^{\prime}, \Omega\right) \hat{y}\left[\left(e_{10}^{y} e_{01}^{y *}\right)\left(X_{2}\left(\underline{r}^{\prime}, \omega_{1},-\omega_{2}\right)+X_{2}\left(\underline{r}^{\prime},-\omega_{2}, \omega_{1}\right)\right)\right]
$$

with $\Omega=\omega_{1}-\omega_{2}$.

At this point, the unknown function $X_{2}$ must be reviewed. Starting from the charge surface density $Q_{y}$ in the non-linear region it can be assumed that this region is a square law device. Thus the charge would be described by the following function:

$$
Q_{y}(\underline{r}, t)=k E_{y}^{2}
$$

where $k$ is an unknown coefficient. Using the expression of Eq. (53) and rewriting the charge $Q_{y}$ in a way similar to Eq. (43):

$$
Q_{y}(\underline{r}, t)=k E_{y}^{2}=k \int_{-\infty}^{+\infty} \mathrm{d} t_{1} \int_{-\infty}^{+\infty} \mathrm{d} t_{2} \delta\left(t-t_{1}\right) \delta\left(t-t_{2}\right) E_{y}\left(\underline{r}, t_{1}\right) E_{y}\left(\underline{r}, t_{2}\right)
$$


the final expression for $X_{2}$ is then denoted:

$$
X_{2}\left(\underline{r}, \omega_{1}, \omega_{2}\right)=k
$$

Substituting Eq. (55) into Eq. (52) the following expression can be derived:

$$
\delta e_{1-1}^{n l}(\underline{r}) \hat{y}=\frac{4 k}{c^{2}} \iiint_{V} \mathrm{~d} V\left(\underline{r^{\prime}}\right) \hat{y} \lim _{\Omega \rightarrow 0} \hat{y}\left(\Omega^{2} \underline{\bar{G}}\left(\underline{r}, \underline{r}^{\prime}, \Omega\right)\right) \hat{y}\left(e_{10}^{y} e_{01}^{y *}\right)
$$

Using the analytical expression of Green's function from [7] the limit of (56) can be calculated in the following:

$$
\lim _{\Omega \rightarrow 0}\left(\Omega^{2} \hat{y} \underline{\bar{G}} \hat{y}\right)=\lim _{\Omega \rightarrow 0} \Omega^{2} \hat{y}\left(\bar{G}_{p r}+\underline{G}_{s e c}\right) \hat{y}=\frac{c^{2}}{4 \pi} \frac{\partial}{\partial y^{2}}\left[\left(\frac{1}{\left|r-r^{\prime}\right|}\right)-\left(\frac{1}{\left|r-r^{\prime \prime}\right|}\right)\right]
$$

Where $r^{\prime \prime}=r^{\prime}-2 z^{\prime} \hat{z}$

Using the expression of (57), Eq. (56) finally becomes:

$$
\delta e_{1-1} \hat{y}=\frac{k}{\pi} \frac{\partial}{\partial y^{2}} \iiint_{V}\left(\frac{1}{\left|r-r^{\prime}\right|}-\frac{1}{\left|r-r^{\prime \prime}\right|}\right)\left(e_{10}^{y} e_{01}^{y *}\right) \mathrm{d} V\left(\underline{r}^{\prime}\right)
$$

Equation (58) is a very simple final expression providing the unknown electrical field of the IF frequency. This solution is connected to the relevant linear problem through the expressions of the $\underline{e}_{10}(\underline{r}), e_{01}^{*}(\underline{r})$ functions. Actually $\underline{e}_{10}(\underline{r})$ is the solution for the electric field of the relevant linear problem at the operation frequency $\omega_{1}$ and $e_{01}^{*}(\underline{r})$ is the solution for the conjugate electric field of the relevant linear problem at frequency $\omega_{2}$. Generalizing this method which has already been presented the electric field of any harmonic $\omega_{n m}$ could be determined provided the relevant linear problems at $n \omega_{1}, m \omega_{2}$ frequencies have already been solved. The only difference will be that the approximation used for $\Omega\left(\omega_{1}-\omega_{2} \stackrel{\omega_{1} \approx \omega_{2}}{\longrightarrow} 0\right)$ will no longer exist and the final Eq. (24) will also have to include a double spectral integral of Green's function. Usually these integrals cannot be solved analytically and numerical methods have to be used. So using [9] the expressions of the $\underline{e}_{10}(\underline{r})$, $e_{01}^{*}(\underline{r})$ functions are the following:

$$
e_{i, 10}^{y}=c_{i, \omega_{1}}^{y} e^{j k_{\omega_{1}}^{i}\left(y^{\prime}-y_{c}^{i}\right)}, \quad e_{i, 01}^{y *}=c_{i, \omega_{2}}^{y *} e^{-j k_{\omega_{2}}^{i}\left(y^{\prime}-y_{c}^{i}\right)}
$$

where index $i$ here denotes the observation block in which the electric field of the linear problem is calculated.

The $c_{i, \omega_{1}}^{y}, c_{i, \omega_{2}}^{y *}$ are the unknown coefficients of the electric field which are calculated numerically in [9] and $k^{i}$ is the relevant propagation constant. Substituting the expressions of the electric fields from Eq. (59) into Eq. (58) we have:

$$
\begin{aligned}
\delta e_{1-1} \hat{y}= & c_{i, \omega_{1}}^{y} c_{i, \omega_{2}}^{y} e^{-j\left(k_{\omega_{1}}^{i}-k_{\omega_{2}}^{i}\right) y_{c}^{i}} \frac{k}{\pi} \frac{\partial}{\partial y^{2}} \iiint_{V}\left(\frac{1}{\left|r-r^{\prime}\right|}\right) e^{j\left(k_{\omega_{1}}^{i}-k_{\omega_{2}}^{i}\right) y^{\prime}} \mathrm{d} V\left(\underline{r}^{\prime}\right) \\
& -c_{i, \omega_{1}}^{y} c_{i, \omega_{2}}^{y} e^{-j\left(k_{\omega_{1}}^{i}-k_{\omega_{2}}^{i}\right) y_{c}^{i}} \frac{k}{\pi} \frac{\partial}{\partial y^{2}} \iiint\left(\frac{1}{\left|r-r^{\prime \prime}\right|}\right) e^{j\left(k_{\omega_{1}}^{i}-k_{\omega_{2}}^{i}\right) y^{\prime}} \mathrm{d} V\left(\underline{r}^{\prime}\right)
\end{aligned}
$$


Calculating the triple volume integral of the non linear region with dimensions $L_{x}^{i}=x_{2}^{i}-x_{1}^{i}$, $L_{Y}^{I}=y_{2}^{i}-y_{1}^{i}$ and $L_{z}^{i}=z_{2}^{i}-z_{1}^{i}$ and the double $y$ derivative, the unknown electric field at the center of the non linear block region with coordinates $\left(x_{c}^{i}, y_{c}^{i}, z_{c}^{i}\right)$ can be easily determined with the use of analytical and numerical methods.

\section{CONCLUSIONS}

In conclusion, a generalized 3-D global analysis technique is presented to treat MMIC structures, using integral equation techniques. The use of Galerkin technique allows the analysis of arbitrary geometries. Numerical results are presented for specific longitudinal discontinuities while several novelties are being implemented including the three dimensional approach, the finite conductivities and the treatment of the incident, reflective and transmitting waves in the input and output microstrip lines of the circuitry.

\section{References}

[1] Katehi, P. B. (1987). A generalized method for the evaluation of mutual coupling in microstrip arrays. IEEE Trans. on Antennas and Propagation, AP-35, 125-133.

[2] Goyal, R. (Ed.) (1989). Monolithic Microwave Integrated Circuits: Technology and Design. Artech House, Boston.

[3] (1993). Modeling and design of coplanar monolithic microwave and millimeter-wave integrated circuits. IEEE Trans. on Microwave Theory and Techniques, 41 (special issue).

[4] Jones, D. S. (1964). Theory of Electromagnetism. Pergamon Press, London.

[5] Collin, R. E. (1960). Field Theory of Guided Waves. McGraw-Hill Co, New York.

[6] Sommerfeld, A. (1949). Partial Differential Equations of Physics. Academic Press, New York.

[7] Athanasoulias, G. B. (1995). Analysis of integrated optical and millimeter wave circuits using entire domain base functions. PhD thesis, NTUA.

[8] Holt, A. R., Uzunoglu, N. K. and Evans, B. G. An integral equation solution to the scattering of electromagnetic radiation by dielectric spheroids and ellipsoids. IEEE Trans. on Antennas and Propagation, AP-26, 706-712.

[9] Makri, R. J. (1999). Modeling, design and development of microwave-millimeter wave integrated circuits and systems for telecommunications applications. PhD thesis, NTUA.

[10] Itoh, T. and Mittra, R. (1973). Spectral-domain approach for calculating the dispersion characteristics of microstrip lines. IEEE Trans. on Microwave Theory and Techniques, MTT-21, 496-499.

[11] Harrington, R. F. (1967). Matrix methods for field problems. Proceedings of the IEEE, 55(2), 136-149.

[12] Neu, M. N. (1985). Method of moments as applied to electromagnetic problems. IEEE Trans. on Microwave Theory and Techniques, MTT-33, 972-980.

[13] Grandinetti, L., Kowalik, J. and Vajtersic, M. (Eds.) (1997). Advances in High Performance Computing, NATO ASI Series, Kluwer Academic Publishers. 

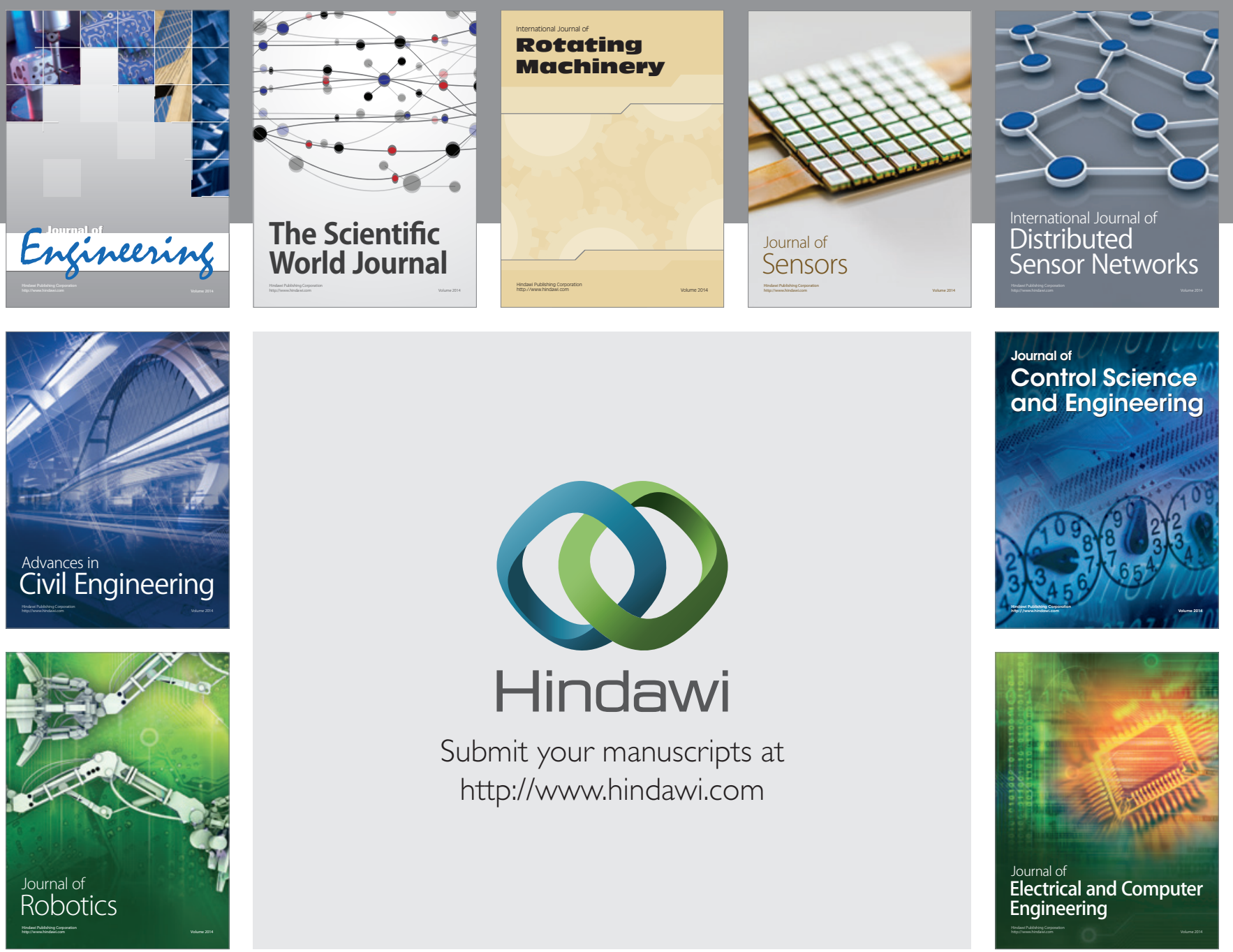

Submit your manuscripts at

http://www.hindawi.com
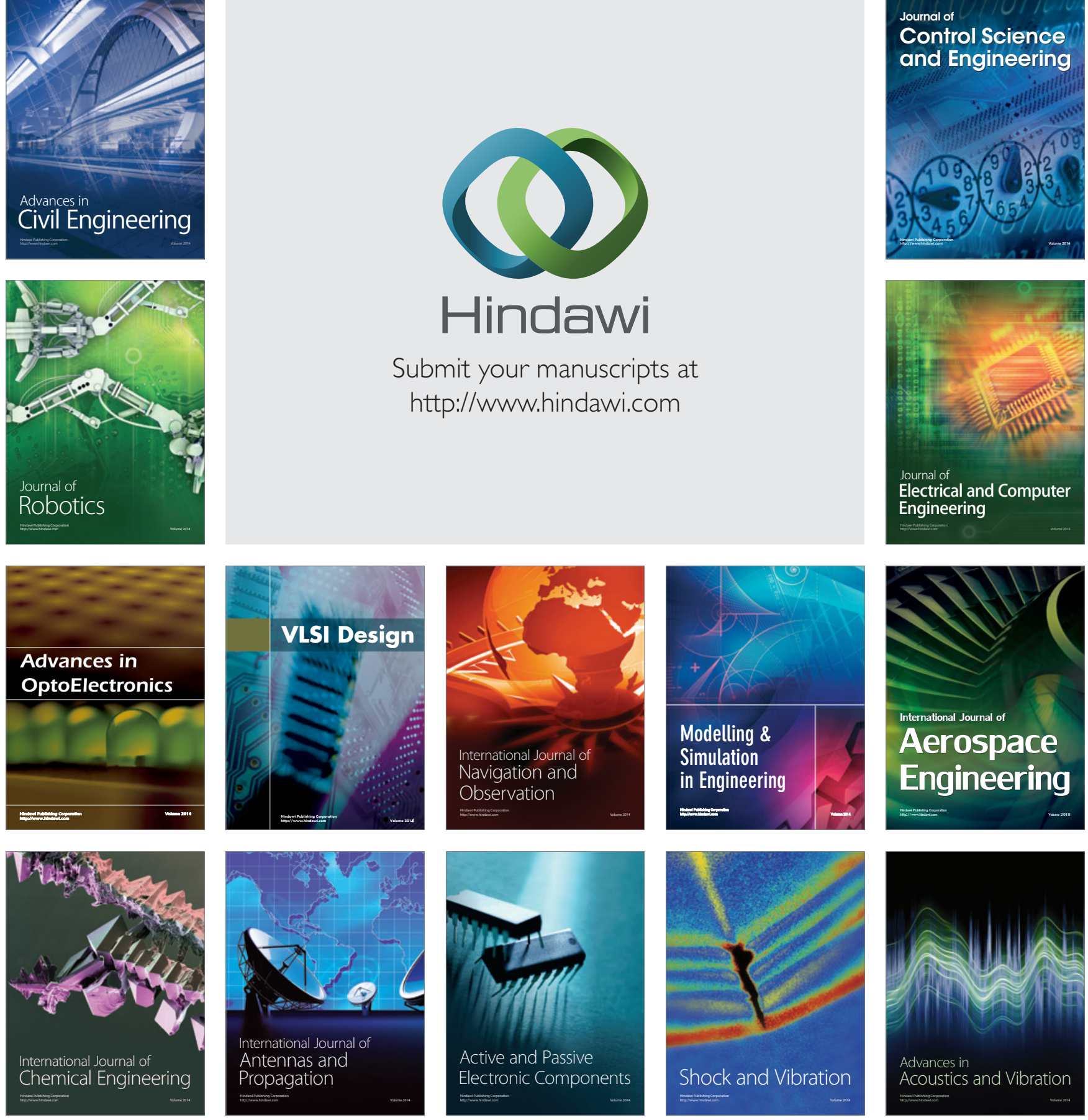